\title{
Global transcriptional profiling of Burkholderia pseudomallei under salt stress reveals differential effects on the Bsa type III secretion system
}

\author{
Pornpan Pumirat1,2, Jon Cuccui2, Richard A Stabler2, Joanne M Stevens 3 , Veerachat Muangsombut 1 , \\ Ekapot Singsuksawat ${ }^{1}$, Mark P Stevens ${ }^{3}$, Brendan W Wren² and Sunee Korbsrisate*1
}

\begin{abstract}
Background: Burkholderia pseudomallei is the causative agent of melioidosis where the highest reported incidence world wide is in the Northeast of Thailand, where saline soil and water are prevalent. Moreover, recent reports indicate a potential pathogenic role for B. pseudomallei in cystic fibrosis lung disease, where an increased sodium chloride ( $\mathrm{NaCl}$ ) concentration in airway surface liquid has been proposed. These observations raise the possibility that high salinity may represent a favorable niche for $B$. pseudomallei. We therefore investigated the global transcriptional response of $B$. pseudomallei to increased salinity using microarray analysis.

Results: Transcriptome analysis of B. pseudomallei under salt stress revealed several genes significantly up-regulated in the presence of $320 \mathrm{mM} \mathrm{NaCl}$ including genes associated with the bsa-derived Type III secretion system (T3SS).

Microarray data were verified by reverse transcriptase-polymerase chain reactions (RT-PCR). Western blot analysis confirmed the increased expression and secretion of the invasion-associated type III secreted proteins BipD and BopE in B. pseudomallei cultures at 170 and $320 \mathrm{mM} \mathrm{NaCl}$ relative to salt-free medium. Furthermore, salt-treated $B$.

pseudomallei exhibited greater invasion efficiency into the lung epithelial cell line A549 in a manner partly dependent on a functional Bsa system.

Conclusions: B. pseudomallei responds to salt stress by modulating the transcription of a relatively small set of genes, among which is the bsa locus associated with invasion and virulence. Expression and secretion of Bsa-secreted proteins was elevated in the presence of exogenous salt and the invasion efficiency was enhanced. Our data indicate that salinity has the potential to influence the virulence of B. pseudomallei.
\end{abstract}

\section{Background}

Burkholderia pseudomallei is a saprophyte and the causative agent of melioidosis, a human infectious disease endemic in some tropical areas including southeast Asia and northern Australia [1]. Inhalation is a recognized route of infection with this organism and pulmonary disease is common [1,2]. Owing to its aerosol infectivity, the severe course of infection, and the absence of vaccines and fully effective treatments, B. pseudomalle $i$ is classified as a hazard category three pathogen and considered a potential biothreat agent [2]. B. pseudomallei, is a Gram negative bacillus found in soil and water over a wide

\footnotetext{
*Correspondence: grsks@mahidol.ac.th

1 Department of Immunology, Faculty of Medicine Siriraj Hospital, Mahidol University, Bangkok 10700, Thailand

Full list of author information is available at the end of the article
}

endemic area and mainly infects people who have direct contact with wet soil $[1,3]$. In Thailand, the highest incidence of melioidosis is in the northeast region, at a rate of approximately $3.6-5.5$ per 100,000 human populations annually. Septicaemic presentation of disease is associated with a high mortality rate (up to $50 \%$ in adults and $35 \%$ in children) [4]. A remaining enigma is that $B$. pseudomallei is commonly present in this region of Thailand, but rarely found in other parts of the country or indeed other parts of the world [5,6]. Of potential significance is the abundance of enclosed bodies of water with a high salt content and saline soils in the northeast region of Thailand [7]. The electrical conductivity of saltaffected soil in Northeast Thailand is ranging between 4 to $100 \mathrm{dS} / \mathrm{m}$, which is higher than normal soil from other 
parts of Thailand (approximately $2 \mathrm{dS} / \mathrm{m}$ ) (Development Department of Thailand). An increase in salt concentration in these regions is believed to be caused both by natural phenomena and man-made activities [7]. One may speculate that the organism has developed an ability to thrive in saline conditions and as such has gained a selective ecological advantage over other soil dwelling micro organisms.

Previously, it has been indicated that the killing efficiency of Burkholderia species, including B. pseudomallei against the nematode Caenorhabditis elegans was enhanced in a high osmolarity conditions [8]. This putative link between high salt concentration and an ability to withstand such conditions is evident in a subset of closely related organisms, namely, the B. cepacia complex (BCC). These are opportunistic pathogens of cystic fibrosis (CF) sufferers $[9,10]$ where the lung airway surface liquid has been hypothesized an increased concentration of $\mathrm{NaCl}$ [11], that is typically 2 -fold higher than in healthy lungs [12]. More recently, reports of a potential pathogenic role for B. pseudomallei in CF lung disease have been made [13]. To date, little is known of how elevated $\mathrm{NaCl}$ concentrations affect $B$. pseudomallei.

As B. pseudomallei can survive and multiply under different environmental conditions and in various hosts $[14,15]$, it is likely that this organism has developed strategies to cope with high salt concentrations in both the natural environment and in its respective hosts. In the river water environment, osmolarity is believed to be less than $60 \mathrm{mM} \mathrm{NaCl}$ whilst in the human lung it is normally 50 to $100 \mathrm{mM}$ and in the blood the bacterium can encounter a concentration of up to $150 \mathrm{mM} \mathrm{NaCl}[11,16]$. Recently, the secreted protein profile of B. pseudomallei following growth in salt-rich medium was revealed and provided a clue to the adaptive response of the organism to this stress [17]. Increased secretion of several metabolic enzymes, stress response protein GroEL, beta-lactamase like proteins and potential virulence factors were noted. Moreover, the effects of increasing salt concentration on the expression of a number of genes within the organism $B$. cenocepacia, formerly B. cepacia genomovar III, a close relative of $B$. pseudomallei have been described [18]. Genes found to be upregulated included an integrase, an NAD-dependent deacetylase and an oxidoreductase amongst others. In Pseudomonas aerugi$n o s a$, another close relative of $B$. pseudomallei, the upregulation of genes associated with osmoprotectant synthesis, putative hydrophilins, and a Type III protein secretion system (T3SS) after growth under steady-state hyperosmotic stress has been demonstrated [19]. High salt stress was also demonstrated to be one of the environmental stimuli affecting expression of the Ysa T3SS in Yersinia enterocolitica $[20,21]$. The B. pseudomallei strain K96243 genome encodes three predicted T3SSs, one related to the Inv/Mxi-Spa systems of Salmonella and Shigella (Bsa, T3SS-3) and two related to systems found in plant bacterial pathogens (T3SS-1 and -2). Of these, only the Bsa system appears to play a significant role in virulence in rodent models of melioidosis [22,23], likely as a consequence of its effects on invasion, endosome escape and intracellular survival $[24,25]$. It is noteworthy that transcription of the invasion-associated Salmonella pathogenicity island-1 genes homologous to the $b s a$ locus is activated by the addition of $\mathrm{NaCl}$ [26]. Gaining an understanding of the ability of $B$. pseudomallei to survive in the presence of high salt concentrations is therefore significant, as this may provide insights into its pathogenicity and persistence in endemic areas.

Here we used a genome-wide oligonucleotide microarray to quantify the transcription of $B$. pseudomallei genes in response to salt stress. Differential regulation of a subset of genes was confirmed by RT-PCR and by analysis of production of the encoded proteins. Our data reveal that exogenous $\mathrm{NaCl}$ induces the virulence-associated Bsa T3SS and the consequences of such for invasion of A549 cells were investigated.

\section{Results}

\section{B. pseudomallei growth was inhibited in high salt}

To better understand the physiology of B. pseudomallei in response to elevated salt, we titrated the effect of salt on B. pseudomallei growth starting from salt-free Luria Bertani (LB) medium and standard LB medium containing $170 \mathrm{mM}$ plus various concentrations of $\mathrm{NaCl}(170+150$, $170+300$ and $170+450 \mathrm{mM}$ ), and found that conditions with 470 and $620 \mathrm{mM} \mathrm{NaCl}$ had severe impairment on $B$. pseudomallei growth (data not shown). For lower $\mathrm{NaCl}$ concentrations, the growth kinetics of $B$. pseudomallei K96243 cultured in standard LB medium containing 170 or $320 \mathrm{mM} \mathrm{NaCl}$ was similar until $6 \mathrm{hrs}$; the growth rate thereafter was impaired when cultured in LB broth containing $320 \mathrm{mM} \mathrm{NaCl}$ (Figure 1). The doubling time in $\mathrm{NaCl}$-supplemented LB broth was calculated to be $53 \pm$ 4.3 min compared to $38 \pm 3.0 \mathrm{~min}$ in standard LB broth ( $t$-test; $P$ value $=0.027)$. In addition, we found that growth of $B$. pseudomallei in salt-free medium was faster than in standard LB medium supplemented with 170 and $320 \mathrm{mM} \mathrm{NaCl}$ (Figure 1). This data indicated that increased $\mathrm{NaCl}$ reduced the logarithmic growth rate of $B$. pseudomallei.

\section{Differential transcriptome of B. pseudomallei during growth in high salt}

Our studies indicated that growth of B. pseudomallei was severely impaired during culture at $\mathrm{NaCl}$ concentrations of 470 and $620 \mathrm{mM}$ (data not shown). This suggested that these concentrations may be too high to detect salt-specific transcriptional changes. A previous study carried 


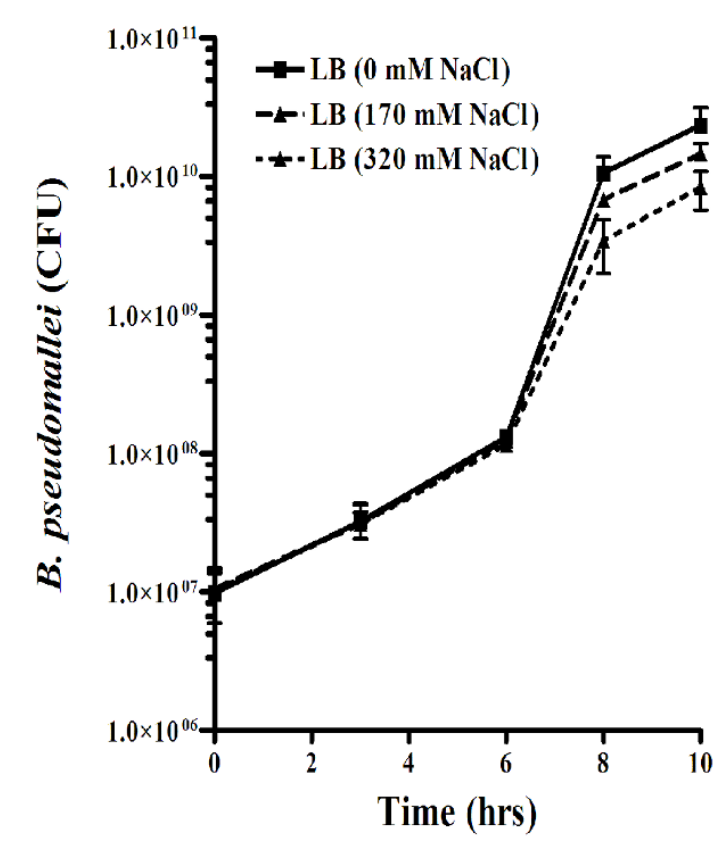

Figure 1 Growth kinetics of B. pseudomallei. B. pseudomallei K96243 growth in LB broth containing 0,170 or $320 \mathrm{mM} \mathrm{NaCl}$ was determined by colony plate counting. The data points and error bars represent mean colony forming unit (CFU) and standard deviation from triplicate experiments.

out in our laboratory demonstrated a significantly altered secretome when the organism was grown in $320 \mathrm{mM}$ $\mathrm{NaCl}$ compared to standard LB medium $(170 \mathrm{mM} \mathrm{NaCl})$ [16]. We therefore chose 170 and $320 \mathrm{mM} \mathrm{NaCl}$ conditions for further investigation by microarray analysis. We elected to isolate RNA from cultures at 3 and 6 hrs for transcriptome analysis because no significant difference in bacterial growth survival was noted at these time points (Figure 1). RNA was stabilized and extracted immediately and analyzed for differential gene expression by hybridisation to a $B$. mallei/pseudomallei whole genome 70 mer oligonucleotide microarray version 2 (a kind gift from the J. Craig Venter Institute) which containing 9,826 reporters based on the B. mallei ATCC 23344, B. mallei GB8 Horse 4, B. pseudomallei $1710 \mathrm{~b}$ and B. pseudomallei K96243 genome. Four biological replicates generated for each sample clustered together indicating minimal experimental variation (Additional file 1). ANOVA statistical analysis and multiple testing correction identified 10 genes as significantly altered in their transcription (Table 1). Among the salt-regulated genes of B. pseudomallei identified in this study were a putative two-component system response regulator, bacterial metabolic enzymes, and hypothetical proteins. Fold changes of altered genes at both 3 and 6 hrs ranged from 1.1-1.8 and 1.1-26.6, respectively. Noticeably, a larger dynamic range of gene expression was observed after 6 hrs cultures, with the majority of the 10 genes being upregulated.
Due to the stringent statistic analysis by ANOVA and false discovery rate correction, it is possible that potentially significant trends were masked. Owing to the effect of salt on loci encoding T3SS in Pseudomonas, Yersinia and Salmonella, we examined the microarray data for effects on predicted Type III secretion-associated loci by only looking at the test ratio and standard deviation (SD) and computing a confidence of that data point using a standard two tailed $t$-test (Table 2). Interestingly, a number of $b s a$-derived T3SS genes were found to have altered expression levels during culture in LB broth containing $320 \mathrm{mM} \mathrm{NaCl}$ compared to standard LB at $3 \mathrm{hrs}$ and $6 \mathrm{hrs}$ ( $t$-test; $P$ value < 0.05$)$ (Table 2 and Additional file 2), in particular those encoding predicted secreted effectors and translocon components. We also found that the expression of beta-lactamase family protein (BPSS2119) and GroEL (BPSS0477) was upregulated in LB containing $320 \mathrm{mM} \mathrm{NaCl}$ by approximately 1.2 fold compared with those in standard LB broth at the $6 \mathrm{hrs}$ time point ( $t$-test; $P$ value $<0.05$ ) (Additional file 3 ). In contrast genes encoding for T3SS-1 and T3SS-2 (except BPSS1603 and BPSS1617) did not show a significant difference in expression levels $(t$-test; $P$ value $>0.05)$ (Additional file $3)$.

By looking at the transcription of bsa-encoded genes, we were able to establish that $\mathrm{NaCl}$ induces their expression. However it is possible that other T3SS effectors encoded elsewhere on the chromosome might be coexpressed with bsa genes in response to salt stress. To find other candidate T3SS effectors of B. pseudomallei, we used Self Organization Maps (SOM) based on the transcription profiles of the genes encoding the effectors BopA and BopE to identify 94 genes that had similar expression patterns (Additional file 4.) Among the coregulated genes were other bsa-associated genes (e.g. those encoding $\mathrm{BipB}$ and the predicted chaperone BicP). Moreover, we also examined the direction and magnitude of transcription of predicted T3SS effectors that were previously proposed by Haraga et al [26] on the basis of homology with known effectors of other bacteria (Table 3 and Additional file 5). The results showed that only the T3SS-associated genes encoded within the bsa locus appeared to be significantly induced under salt stress (bopA, bopE, bipC, bipB, bsaP), with non-Bsa putative effectors apparently being insensitive to exogenous $\mathrm{NaCl}$ under the conditions tested. Thus, we did not find any other candidate T3SS effectors among the genes co-regulated with BopA and BopE, including those identified recently by Haraga et al. [27].

\section{Validation of the differential transcription of $B$. pseudomallei genes by exogenous salt}

To validate the differential transcription of genes observed by microarray analysis, selected transcripts were amplified by RT-PCR and band intensities quantified by densitometric analysis. The experiments were 
Table 1: Effect of NaCl treatment on transcription of B. pseudomallei K96243 genes as detected by microarray analysis.

\begin{tabular}{|c|c|c|c|c|}
\hline \multirow[t]{2}{*}{ Putative function } & \multirow[t]{2}{*}{ Gene } & \multicolumn{2}{|c|}{ Fold change } & \multirow[t]{2}{*}{ Pvalue } \\
\hline & & 3 hrs & $6 \mathrm{hrs}$ & \\
\hline Formyltetrahydrofolate deformylase & BPSL0543 & $1.3^{*}$ & -1.1 & 0.037 \\
\hline Putative adenylate cyclase & BPSL3054 & $1.5^{*}$ & -1.0 & 0.038 \\
\hline Acyl-CoA dehydrogenase domain protein & BPSS1272 & 1.0 & $4.4^{*}$ & 0.035 \\
\hline Hypothetical protein & BPSS2215 & -1.2 & $7.3^{*}$ & 0.038 \\
\hline Hypothetical protein & BPSS2221 & 1.0 & $3.0^{*}$ & 0.037 \\
\hline Response regulator & BPSS2231 & -1.4 & $6.4^{*}$ & 0.038 \\
\hline Hypothetical protein & BPSS2232 & 1.1 & $26.6^{*}$ & 0.037 \\
\hline Hypothetical protein & BPSS2240 & -1.8 & $6.8^{*}$ & 0.038 \\
\hline Short chain dehydrogenase/oxidoreductase & BPSS2242 & 1.0 & $10.0^{*}$ & 0.035 \\
\hline Glycosyltransferase family 9 protein & BPSS2255 & 1.0 & $2.6^{*}$ & 0.037 \\
\hline
\end{tabular}

* Genes showed mean significant differences comparing between standard LB medium (170 mM) and LB with $320 \mathrm{mM} \mathrm{NaCl} \mathrm{using} \mathrm{ANOVA}$ with a Benjamini-Hochberg multiple testing correction ( $P$ value $<0.05)$.

performed in duplicate using total RNA extracted from bacteria grown in salt-free LB, standard LB $(170 \mathrm{mM}$ $\mathrm{NaCl}$ ) and $\mathrm{LB}$ containing $320 \mathrm{mM} \mathrm{NaCl}$ at 3 and $6 \mathrm{hrs}$ post-inoculation. In all cases, RT-PCR analysis mirrored the timing and direction of change of transcription of the differentially transcribed genes identified by microarray analysis (Figure 2). In most cases the magnitude of the change was also comparable. Thus, up-regulation of BPSS2232, BPSS1272 and BPSS2242 (which respectively encode an Acyl-CoA dehydrogenase, a hypothetical protein and an oxidoreductase) was confirmed to occur at 6 hrs but not $3 \mathrm{hrs}$ in the presence of added $\mathrm{NaCl}$ as found by microarray analysis (Table 1 ). Furthermore, the $b s a$ derived genes BPSS1529, BPSS1524, and BPSS1525 (which respectively encode the translocon component BipD and effectors BopA and BopE) were confirmed by RT-PCR to be upregulated in the presence of $320 \mathrm{mM}$ $\mathrm{NaCl}$ (Figure 2). Increases for the $b s a$-derived genes occurred in a dose dependent manner, increasing from zero to $170 \mathrm{mM}$ to $320 \mathrm{mM} \mathrm{NaCl}$ (Figure 2).

The finding that genes encoding the Bsa T3SS were induced under high salinity was also reflected in protein levels. When B. pseudomallei K96243 was cultured in LB broth containing $320 \mathrm{mM} \mathrm{NaCl}$, expression and secretion of the invasion-associated Type III secreted proteins BipD and BopE was enhanced when compared to standard LB, and in turn levels were higher than in salt-free medium (Figure 3). We observed a correlation between the increased expression of BopE and BipD from almost salt-free medium to higher levels of salt suggesting the importance of salt in the induction of the T3SS. These patterns of induction were also noted in an independent B. pseudomallei strain designated 10276 (data not shown) [28]. Taken together, these findings imply that expression of the Bsa T3SS of B. pseudomallei is enhanced by salt stress.

\section{Salt-stress increases invasion of host cells by B. pseudomallei}

The ability of $B$. pseudomallei to invade non-phagocytic host cells is partly dependent on the Bsa T3SS [1,2] and is believed to contribute to the pathogenesis of melioidosis. Owing to the induction of $b s a$ genes by exogenous salt, we investigated whether salt stress affects invasion of $B$. pseudomallei into A549 human lung respiratory epithelial cells. Overnight culture of B. pseudomallei in LB broth supplemented with $\mathrm{NaCl}$ (170 and $320 \mathrm{mM}$ ) led to significantly increased invasion into A549 cells relative to bacteria cultured in $\mathrm{NaCl}$-free $\mathrm{LB}$ broth $(P$ value $=0.0002$ and 0.0022 , respectively) (Figure 4 ). We additionally showed a significant difference in invasion capacity between B. pseudomallei cultured in LB with 170 and 320 $\mathrm{mM} \mathrm{NaCl}(P$ value $=0.0272)$. The invasion efficiency of B. pseudomallei grown in $\mathrm{NaCl}$-free LB was $0.09 \%$ in contrast to, those of salt-treated bacteria $(0.49$ and $0.88 \%$ in LB with 170 and $320 \mathrm{mM} \mathrm{NaCl}$, respectively). To our knowledge this is the first report revealing that salinity affects the ability of $B$. pseudomallei to invade host cells. Although invasion was enhanced after overnight culture in salt-containing media, culturing B. pseudomallei in $\mathrm{NaCl}$ supplemented medium up to $320 \mathrm{mM}$ for either 3 
Table 2: Effect of $\mathrm{NaCl}$ on transcription of genes associated with the bsa-derived T3SS in B. pseudomallei K96243.

\begin{tabular}{|c|c|c|c|}
\hline \multirow[t]{2}{*}{ Putative function } & \multirow[t]{2}{*}{ Gene } & \multicolumn{2}{|c|}{ Fold change } \\
\hline & & $3 \mathrm{hrs}$ & $\begin{array}{c}6 \\
\text { hrs }\end{array}$ \\
\hline \multicolumn{4}{|l|}{$\begin{array}{c}\text { Type III structural } \\
\text { proteins }\end{array}$} \\
\hline BsaZ & BPSS1534 & 1.3 & -1.0 \\
\hline BsaY & BPSS1535 & $2.3^{*}$ & 1.3 \\
\hline BsaX & BPSS1536 & 1.2 & -1.2 \\
\hline BsaW & BPSS1537 & $1.2^{*}$ & 1.2 \\
\hline BsaV & BPSS1538 & 1.1 & 1.1 \\
\hline BsaU & BPSS1539 & $2.9^{*}$ & 1.0 \\
\hline Bsat & BPSS1540 & $1.6^{*}$ & $1.9^{*}$ \\
\hline BsaS & BPSS1541 & $1.6^{*}$ & 1.2 \\
\hline BsaR & BPSS1542 & 1.1 & 1.1 \\
\hline BsaQ & BPSS1543 & 1.2 & 1.1 \\
\hline BsaP & BPSS1544 & $2.4^{*}$ & 1.1 \\
\hline BsaO & BPSS1545 & 1.3 & 1.1 \\
\hline BsaN & BPSS1546 & 1.3 & 1.1 \\
\hline Bsal & BPSS1548 & -1.1 & 1.3 \\
\hline BsaK & BPSS1549 & 1.1 & 1.2 \\
\hline \multicolumn{4}{|l|}{ Translocator proteins } \\
\hline BipD & BPSS1529 & $1.8^{*}$ & $1.8^{*}$ \\
\hline BipC & BPSS1531 & $1.4^{*}$ & $1.4^{*}$ \\
\hline BipB & BPSS1532 & 1.3 & 1.3 \\
\hline \multicolumn{4}{|l|}{ Effector proteins } \\
\hline ВорВ & BPSS1517 & -1.2 & 1.0 \\
\hline BopA & BPSS1524 & $2.2^{*}$ & 1.8 \\
\hline BopE & BPSS1525 & 1.2 & $1.4^{*}$ \\
\hline
\end{tabular}

* Genes showed mean significant differences comparing between standard LB medium (170 mM) and LB with $320 \mathrm{mM}$ $\mathrm{NaCl}$ using $t$-test $(P$ value $<0.05)$.

or 6 hrs did not significantly affect the ability of the bacteria to invade A549 cells (data not shown).

\section{Discussion}

Alterations in $\mathrm{NaCl}$ content and therefore osmolarity in various environmental and host conditions are known conditions that most bacteria must counteract for survival [16]. At low concentrations, $\mathrm{NaCl}$ is necessary for bacterial growth, however at high concentrations it is capable of causing considerable stress and even cell death. B. pseudomallei is an environmental saprophyte that can survive and multiply under difficult environmental conditions [1,2]. It is likely therefore that B. pseudomallei must have the mechanisms to sense changes in osmolar- ity in the environment and host, and to modulate its gene expression accordingly.

We found that at high salt concentration $(320 \mathrm{mM}$ final concentration of $\mathrm{NaCl}$ ), there was no significant impairment in B. pseudomallei growth over a $6 \mathrm{hr}$ period. This finding is consistent with observations in $B$. cenocepacia indicating that it can tolerate medium containing up to $450 \mathrm{mM} \mathrm{NaCl}$ for $10 \mathrm{hrs}$ [18]. In our study, two and eight genes were shown to be significantly up-regulated in $B$. pseudomallei grown in high salt for 3 and 6 hrs respectively, when compared with standard LB medium containing $170 \mathrm{mM} \mathrm{NaCl}$. Of the 10 genes that show a saltinduced increase in transcription, 7 are clustered on chromosome 2, which is enriched in genes mediating $B$. pseudomallei adaptation and virulence [29]. Importantly, none of these genes were among the list of growth phaseregulated genes identified by microarray analysis of $B$. pseudomallei by Rodrigues et al [30]. This implies that the altered transcription levels detected in this study are a reflection of the salt stress and not impairment of growth.

Although highly stringent statistical analysis identified only a small number of transcriptionally salt-altered $B$. pseudomallei genes, our data did correlate with previous findings in other bacteria. Remarkably, it has been reported that an adenylate cyclase $(\mathrm{CyaB})$ acts as an osmosensor in the Gram negative saprophytic bacterium Myxococcus xanthus [31]. We found a 1.5 fold increase in the expression of a B. pseudomallei K96243 adenylate cyclase gene (BPSL3054) during exposure to high salt for $3 \mathrm{hrs}$ which decreased again later. We postulate therefore that adenylate cyclase might function as an osmosensor in B. pseudomallei, or be involved in the transmission of the signal. For the formyltetrahydrofolate deformylasederived gene (BPSL0543) that was also upregulated at 3 hrs may function in the same manner. In addition, another study by Bhatt and Weingart [18] reported that an oxidoreductase encoding gene $(b s r A)$ has been found to be regulated in response to increasing $\mathrm{NaCl}$ concentrations in B. cenocepacia. A putative oxidoreductase encoding gene (BPSS2242) in B. pseudomallei K96243 was also up-regulated (10 fold up at $6 \mathrm{hrs)}$ under salt stress. However, the exact role that oxidoreductases play in adaptation to osmotic stress is still unknown.

A study into the salt stress response of Azospirillum brasilense, a Gram-negative nitrogen-fixing bacterium associated with various plants, found an increase in the expression levels of its Acyl-CoA dehydrogenase coding gene [32]. Several reports indicate that Acyl-CoA dehydrogenases are involved in the changes of bacterial membrane fluidity during salt tolerance [33,34]. Our study identified an increased level of expression of BPSS1272 also coding for Acyl-CoA dehydrogenase domain protein (around 4.4 fold at $6 \mathrm{hrs}$ ) suggesting that Acyl-CoA dehydrogenase may play a role in response to high salt stress. 
Table 3: Effect of $\mathrm{NaCl}$ on transcription of genes associated with homologs of known T3SS effectors in B. pseudomallei K96243 .

\begin{tabular}{|c|c|c|c|}
\hline \multirow[t]{2}{*}{ Putative function } & \multirow[t]{2}{*}{ Gene } & \multicolumn{2}{|c|}{ Fold change } \\
\hline & & 3 hrs & 6 hrs \\
\hline FG-GAP/YD repeat domain protein & BPSL0590 & -1.2 & -1.1 \\
\hline DNA polymerase III subunits gamma and tau & BPSL1498 & 1.2 & 1.0 \\
\hline Putative outer membrane protein & BPSL1631 & -1.1 & 1.3 \\
\hline Hypothetical protein & BPSL1705 & -1.0 & 1.0 \\
\hline Putative lipoprotein & BPSL1902 & -1.2 & -1.0 \\
\hline RND efflux system, outer membrane lipoprotein, NodT family protein & BPSL1972 & 1.2 & -1.1 \\
\hline Putative exported phospholipase & BPSL2198 & -1.0 & 1.1 \\
\hline Putative methyl-accepting chemotaxis protein & BPSL2367 & -1.6 & 1.0 \\
\hline Putative prolin-rich exported protein & BPSL2472 & -1.2 & -1.1 \\
\hline Hypothetical protein & BPSL2699 & -1.1 & 1.2 \\
\hline Hypothetical protein & BPSS0088 & 1.3 & -1.1 \\
\hline Pentapeptide repeat family protein & BPSS0182 & 1.0 & 1.0 \\
\hline Hypothetical protein & BPSS0183 & -1.1 & 1.2 \\
\hline Surface-exposed protein & BPSS0796 & 1.0 & 1.1 \\
\hline ATP/GTP binding protein & BPSS1385 & -1.2 & 1.0 \\
\hline Tash protein PEST motif family & BPSS1434 & -1.1 & -1.0 \\
\hline Membrane-anchored cell surface protein & BPSS1439 & -1.1 & -1.0 \\
\hline Hypothetical protein & BPSS1504 & 1.2 & 1.3 \\
\hline Hypothetical protein & BPSS1505 & 1.1 & 1.1 \\
\hline BopA & BPSS1524 & 2.2 & 1.8 \\
\hline BopE & BPSS1525 & 1.2 & 1.4 \\
\hline BipC & BPSS1531 & 1.4 & 1.4 \\
\hline ВipB & BPSS1532 & 1.3 & 1.3 \\
\hline BsaP & BPSS1544 & 2.4 & 1.1 \\
\hline Putative lipoprotein & BPSS1974 & -1.0 & 1.1 \\
\hline Hypothetical protein & BPSS2063 & -1.1 & 1.1 \\
\hline Hypothetical protein & BPSS2166 & 1.0 & -1.2 \\
\hline
\end{tabular}

We hypothesise that this role may be in modulation of the membrane layer when $B$. pseudomallei encounters high salt.

As osmotic shock was found to increase expression of T3SS in various pathogens [19-21], we also sought to obtain information on the effect of salt on transcription of the T3SSs of B. pseudomallei. Much research has been carried out on the Bsa T3SS of B. pseudomallei, demonstrating its critical role in pathogenesis and more precisely in escaping the phagosome $[24,28,35]$, but few substrates secreted by this system have been identified $[28,35]$. We used a two tailed unpaired $t$-test to identify genes significantly up-regulated at $3 \mathrm{hrs}$. Our finding that the $b s a$-derived genes, in particular those encoding secreted translocon and effector proteins, are upregulated in the presence of salt by both microarray and RTPCR analysis mirrors the ability of exogenous $\mathrm{NaCl}$ to activate T3SS in other bacteria. T3SS genes encoding for structural components, translocators and effectors in $P$. aeruginosa were upregulated under steady-state hyperosmotic stress [19], as were Salmonella Typhimurium SPI-1 genes encoding T3SS-1 translocon proteins in the presence of exogenous $\mathrm{NaCl}$ [26]. Interestingly, by $t$-test we also found that $B$. pseudomallei grown in high salt upregulated genes encoding a beta-lactamase family protein (BPSS2119) and GroEL (BPSS0477). The increased expression of these genes correlates with the report of increased beta-lactamase family and GroEL proteins 


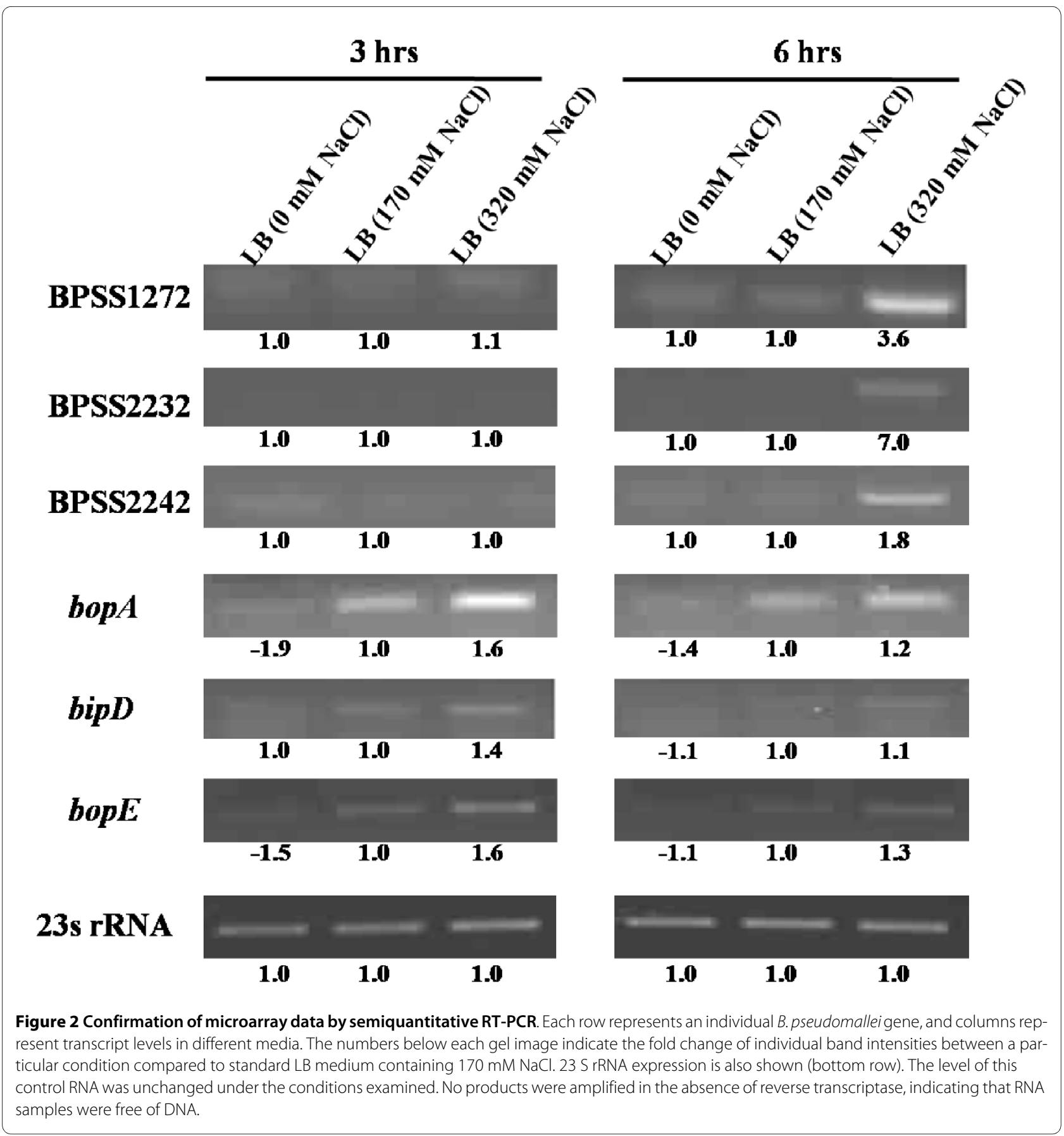

detection in the $B$. pseudomallei secretome under high salinity [17]. Conversely, none of $B$. pseudomallei genes encoded for within T3SS-1, T3SS-2, and other virulence factors (i.e., phospholipases, hemolysin and Burkholderia intracellular motility A) were altered under salt stress in our study (Additional file 3 ).

Previously, Moore et al. [36] demonstrated a functional link between the ability to assimilate L-arabinose and repression of the $b s a$-derived Type III secretion genes, which the authors found may account for the differential virulence of ara-plus and -minus biotypes. Moore et al. [36] also analysed the global transcriptome of B. pseudomallei in the presence or absence of the ara operon to identify genes that may be co-regulated with the $b s a$ apparatus. It is noteworthy that $b s a N$, a predicted positive transcriptional regulator of the $b s a$ genes is up-regulated 1.3 fold at $3 \mathrm{hrs}$ in $\mathrm{NaCl}$-supplemented medium (though not significant by $t$-test), and further studies will be required to unravel the role of $b s a N$ and other regulators in salt induction of T3SS genes. 


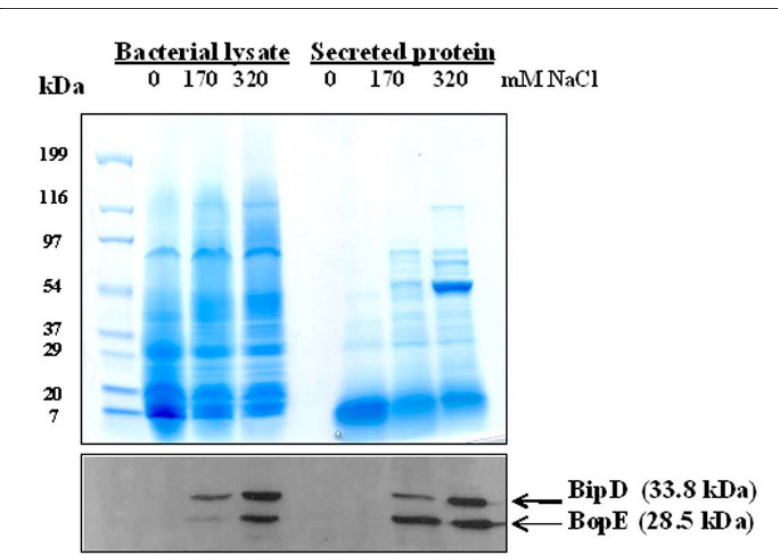

Figure 3 BipD and BopE expression of B. pseudomallei cultured in LB medium with and without exogenous salt. B. pseudomallei K96243 was cultured in LB broth supplemented with 0, 170, or 320 mM $\mathrm{NaCl}$ for 6 hrs. Bacterial lysate and secreted proteins were separated by $12 \%$ polyacrylamide gel and the blotted proteins were reacted with an anti-BipD and anti-BopE antibodies as described in the Methods. Molecular mass markers are shown on the left. Lanes 1-3 are bacterial cell lysates and lanes 4-6 are secreted proteins from culture supernatants.

A recent study generated a list of putative T3SS effectors in B. pseudomallei by comparing predicted coding sequences to known bacterial effectors including Salmonella and Shigella effector proteins [27]. Our investigation could not detect the co-regulation of these putative

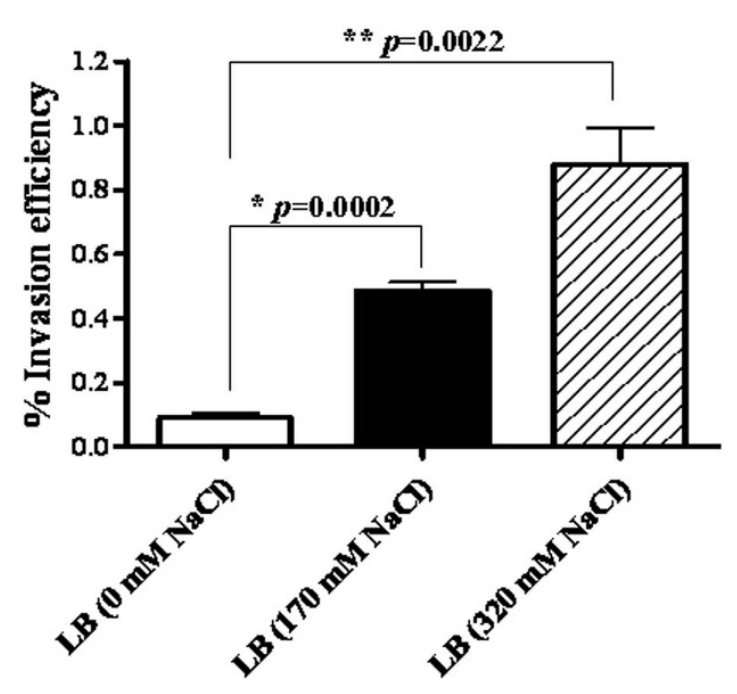

Figure 4 Invasion of A549 epithelial cells by B. pseudomallei. A549 cells were infected with an overnight cultures of B. pseudomallei K96243 grown in in NaCl-free LB broth (open bar), LB broth with 170 $\mathrm{mM} \mathrm{NaCl}$ (solid bar), or LB broth with $320 \mathrm{mM}$ (striped bar). Intracellular bacteria were counted after lysing infected cells at $4 \mathrm{hrs}-$ post-infections. Asterisks indicate significant differences ( $P$ value $<0.05, t$-test) between groups. Error bars represent standard errors of the means for experiments performed in triplicate. effector genes, such as a putative proline-rich exposed protein and ATP/GTP binding protein, with respect to salt stress in contrast to secreted effectors encoded within the bsa locus.

In an attempt to identify genes that may be co-regulated with the virulence-associated Bsa system under salt stress, we used Self Organization Maps based on BopA and BopE expression to find 94 genes with similar expression patterns. These transcriptional changes showed an up-regulation of genes associated with various bacterial functions not only T3SS but also metabolism, stress response, and membrane transportation. One of these genes was the bsa T3SS translocator bipB, which is involved in B. pseudomallei survival within macrophages [35]. Likewise, we also found the up-regulation of the RpoE regulatory gene, $m u c B$. The sigma factor E (RpoE) has previously been reported to play a role in the response to environmental stress tolerance such as hyperosmolarity in B. pseudomallei [37]. Recently, it has been suggested that RpoE and AlgR in P. aeruginosa may coordinate regulation of the T3SS and the alginate biosynthesis pathway [38]. Such a link between RpoE-regulating MucB and salt-induction of the Bsa system may exist in $B$. pseudomallei, but further studies will be required to investigate this.

The salt-induced transcription of the invasion- and virulence-associated genes bipD and bopE, which respectively encode a translocon component [24] and a guanine nucleotide exchange factor that subverts actin dynamics [28], was confirmed to result in increased production and secretion of the proteins by Western blotting using specific antisera. BipD and BopE protein expression increased in a gradient from $0 \mathrm{mM}$ to $170 \mathrm{mM}$ to $320 \mathrm{mM}$ $\mathrm{NaCl}$ at both RNA and protein levels at both 3 and 6 hrs. This provides compelling evidence that the two genes are regulated by $\mathrm{NaCl}$ concentration. BipD and BopE both contribute to invasion of non-phagocytic cells $[24,28]$ and mutation of bipD markedly impairs the virulence of $B$. pseudomallei following intranasal or intraperitoneal inoculation of inbred mice [22]. Consistent with induction of these genes, invasion of A549 cells was enhanced following overnight culture in salt-supplemented LB medium in a manner partly dependent on a functional Bsa system. Likewise, it has been reported in Pseudomonas aeruginosa under steady-state growth that high salt could induce the T3SS [18]. Therefore, it is possible that an overnight culture of $B$. pseudomallei could induce the T3SS and other factors that might contribute in increase invasion efficiency. Our result is in good agreement with a previous report that $S$. typhi cultured in $300 \mathrm{mM} \mathrm{NaCl}$ containing LB broth exhibited an increased secretion of invasion proteins (SipC, SipB and SipA) (Zhao L et al., 2001). Also, this salt-treated S. typhi became highly inva- 
sive toward both epithelial cells and $M$ cell of rat Peyer's pathches (Zhao L et al., 2001).

\section{Conclusions}

This study revealed that B. pseudomallei responds to high salt/osmolarity by modulating the transcription of specific genes. Most of identified genes are within chromosome 2. Among these are several loci that are known to contribute to the pathogenesis of melioidosis, including the invasion-associated Bsa T3SS.

\section{Methods}

\section{Bacterial strains and growth kinetics}

B. pseudomallei strain K96243 was cultured in LB broth at $37^{\circ} \mathrm{C}$ for $18 \mathrm{hrs}$. To determine B. pseudomallei growth kinetics under salt stress, optical density of cultures at various time points was recorded. In brief, overnight-cultured B. pseudomallei adjusted to $\mathrm{OD}_{600} 0.5$ was subcultured 1:500 into standard LB broth without or with supplementation of $\mathrm{NaCl}$ (Merck) to obtain a final concentration of 320-620 mM NaCl. Every 2 hrs after subculture, serial dilution was performed for colony forming unit counts (CFU).

\section{RNA preparation and microarray analysis}

An overnight culture of B. pseudomallei $\mathrm{K} 96243$ was subcultured 1:10 into $10 \mathrm{~mL}$ LB broth containing 170 or 320 $\mathrm{mM} \mathrm{NaCl}$. Four biological replicates were generated and analysed. RNA was isolated from 3 and 6 hrs cultures of B. pseudomallei grown at $37^{\circ} \mathrm{C}$ by adding two volumes of RNAprotect bacterial reagent (QIAGEN) to one volume of bacterial culture and incubating for $5 \mathrm{~min}$ at room temperature. Subsequently, total RNA was extracted from bacterial pellets using Trizol (Invitrogen) according to the manufacturer's instructions and treated with DNase before use.

RNA (Cy3) and B. pseudomallei K96243 genomic DNA (Cy5) labeling were carried out as described in the standard RNA vs DNA labeling protocol [39]. After removal of excess dyes, labelled cDNA was competitively hybridized to B. mallei/pseudomallei microarrays version 2 (kindly supplied by the J. Craig Venter Institute) using a hybridization buffer containing 50\% formamide (Sigma), 5× SSC (Ambion), 0.1\% SDS (Ambion), and $0.1 \mathrm{mM}$ Dithiothreitol solution (DTT) (Sigma) for $20 \mathrm{hrs}$ at $42^{\circ} \mathrm{C}$. After hybridization, the slide was gently agitated in prewarmed $55^{\circ} \mathrm{C}$ low stringency wash solution $(2 \times \mathrm{SSC}, 0.1 \%$ SDS, and $0.1 \mathrm{mM} \mathrm{DTT)}$ and immersed in a new prewarmed $55^{\circ} \mathrm{C}$ low stringency wash solution. Slides were further washed twice in medium stringency wash solution $(0.1 \times$ SSC, $0.1 \%$ SDS, and $0.1 \mathrm{mM}$ DTT). Finally, the slides were washed twice in high stringency wash solution $(0.1 \times$ SSC and 0.1 DTT $)$ and immersed several times in MilliQ/DI water before being allowed to spin dry. The washed slides were scanned using a GMS 418 Array Scanner (Genetic MicroSystems) and fluorescence was quantified using ImaGene v7.5 software (BioDiscovery). Analysis was carried out as previously described [39]. Each time point was normalized to the expression in LB broth without $\mathrm{NaCl}$ prior testing with statistical analysis.

\section{RT-PCR}

The RNA extracts used in the microarray experiments were used to confirm the results obtained from microarray studies using the SuperScript III one-step RT-PCR system (Invitrogen). All genes were amplified using gene

Table 4: Oligonucleotide primers used for RT-PCR.

\begin{tabular}{lll}
\hline Primer Names & Oligo Sequences (5'-3') & Purpose \\
\hline BPSS2232 F & CGGACTTCGACACCGACGCGCTGA & Forward primer for BPSS2232 \\
BPSS2232 R & CGTGTGCAGTCGCTGCCCGCGTA & Reverse primer for BPSS2232 \\
BPSS1272 F & GGCACGAAGGAGTCATCAA & Forward primer for BPSS1272 \\
BPSS1272 R & CGACGCAGTATCTCCAGCTC & Reverse primer for BPSS1272 \\
BPSS2242 F & GTGAGCCGCTACGAGGAC & Forward primer for BPSS2242 \\
BPSS2242 R & ACGCCCCAGTAGTTCGTATC & Reverse primer for BPSS2242 \\
BopA F & GTATTCGGTCGTGGGAATG & Forward primer for bopA \\
BopA R & GCGATCGAAATGCTCCTTAC & Reverse primer for bopA \\
BipD F & GGACTACATCTCGGCCAAAG & Forward primer for bipD \\
BipD R & ATCAGCTTGTCCGGATTGAT & Reverse primer for bipD \\
BopE F & CGGCAAGTCTACGAAGCGA & Forward primer for bopE \\
BopE R & GCGGCGGTATGTGGCTTC G & Reverse primer for bopE \\
$23 S \mathrm{~F}$ & TTCCCGCTTAGATGCTTT & Forward primer for 23S $r$ RNA \\
$23 S \mathrm{R}$ & AAAGGTACTCTGGGGATAA & Reverse primer for 23S rRNA \\
\hline
\end{tabular}


specific primer pairs (Table 4) using the following conditions: $95^{\circ} \mathrm{C}$ (for $45 \mathrm{~s}$ ), $58^{\circ} \mathrm{C}$ (for $45 \mathrm{~s}$ ), and $72^{\circ} \mathrm{C}$ (for $30 \mathrm{~s}$ ) for 25 cycles. Amplification of the $23 \mathrm{~S}$ rRNA gene using $23 \mathrm{~s} \mathrm{~F}$ and $23 \mathrm{~s}$ R primers (Table 4) was included as a control. The experiments were performed in duplicate and analyzed for band intensity by densitometry using GeneSnap/GeneTools software (Syngene).

\section{Preparation of total and secreted protein and Western blotting}

An overnight-culture of B. pseudomallei grown in saltfree LB broth, was centrifuged and the bacteria washed in salt-free medium to remove secreted proteins. The $\mathrm{OD}_{600}$ was adjusted to 0.5 then the washed bacteria subcultured $1: 10$ into LB broth containing 0,170 or $320 \mathrm{mM} \mathrm{NaCl}$ and incubated at $37^{\circ} \mathrm{C}$ for $6 \mathrm{hrs}$. After centrifugation, bacterial pellets were lysed with Laemmli buffer to release intracellular proteins. Secreted proteins were isolated from identical volumes of $0.45 \mu \mathrm{M}$-filtered supernatants from the centrifuged cultures by using Strataclean beads (Stratagene). The supernatants were confirmed to derive from cultures containing identical numbers of viable bacteria, therefore protein levels are not anticipated to reflect cell lysis. Proteins were separated by SDS polyacrylamide gel electrophoresis and transferred to PVDF membrane. The blotted membranes were probed with rabbit polyclonal antiserum against BopE [28] or BipD [24] and detected by horseradish peroxidase-conjugated donkey anti-rabbit IgG (GE Healthcare) and developed using a chemiluminescent substrate (ECL; GE Healthcare).

\section{Invasion assay}

An invasion assay in the human respiratory epithelial cell line A549 was performed as described [25] with some modifications. Briefly, an A549 cell line was infected with overnight culture of B. pseudomallei in LB broth containing 0,170 or $320 \mathrm{mM} \mathrm{NaCl}$ at a multiplicity of infection (MOI) of 50 for $3 \mathrm{hrs}$ to bring bacteria in contact with the cells and allow bacterial entry. The monolayers were overlaid with a medium containing $250 \mu \mathrm{g} / \mathrm{ml}$ of kanamycin (Gibco) to kill extracellular bacteria for $1 \mathrm{hr}$. The viable intracellular bacteria were released from the infected cells at 4 hrs post-infection by lysis with $0.5 \%$ Triton X100 (Sigma-Aldrich) and plated on Trypticase soy agar. Colony forming units were measured after $36-48 \mathrm{hrs}$ of incubation at $37^{\circ} \mathrm{C}$. The percentage invasion efficiency is calculated as the number of intracellular bacteria at $4 \mathrm{hrs}$ post-infection divided by the CFU added $\times 100$. All assays were conducted in triplicate and data from two independent experiments is presented.

\section{Statistical analysis}

In the microarray analysis, the effect of salt on the magnitude of transcription of genes relative to control was tested for statistical significance using ANOVA with a 5\% confidence interval and Benjamini-Hochberg multiple testing correction in GeneSpring (Silicon Genetics). Alternatively, an unpaired $t$-test was calculated for selected-gene groups at the $5 \%$ confidence interval in GraphPad Prism 4 program (Statcon). Results were considered significant at a $P$ value of $\leq 0.05$.

\section{Microarray data accession number}

The complete microarray data set generated in this study is deposited for public access in the ArrayExpress under accession number E-MEXP-2302.

\section{Acknowledgements}

This work was partially supported by the Defense Science and Technology Laboratory (UK) and the Siriraj Grant for Research and Development (Thailand). PP was supported by Siriraj Graduate Scholarship and by the Royal Golden Jubilee Ph.D. Program (PHD0175/2548). We acknowledge the J. Craig Venter Institute for provision of B. pseudomallei/mallei microarrays.

\section{Additional material}

\section{Additional file 1 Cluster diagram of sample replicates in this study. Standard correlation scores between microarray pairs are shown in white. Additional file 2 The effect of $\mathrm{NaCl}$ on transcription of $b s a \mathrm{~T} 3 \mathrm{SS}$ genes in B. pseudomallei K96243 (presented in color graph) \\ Additional file 3 Effect of $\mathrm{NaCl}$ on transcription of selected genes associated with the T3SS-1, T3SS-2, and other virulence/non-viru- lence factors in B. pseudomallei K96243. \\ Additional file $\mathbf{4}$ Ninety four genes identified using Self organization maps (SOM) showed expression patterns similar to bopA and bopE levels. \\ Additional file 5 Effect of $\mathrm{NaCl}$ on transcription of genes encoding homologs of known T3SS effectors in B. pseudomallei K96243 (pre- sented in color graph)}

\section{Authors' contributions}

PP and SK designed the research. PP and ES prepared the DNA/RNA samples for microarray and RT-PCR experiments. PP, RAS and JC carried out the microarray experiment and analysis. JMS performed the Western blotting. PP and VM carried out the invasion assay. PP, JC and SK wrote the manuscript. MPS and BWW critically revised the manuscript for its important intellectual content. All authors read and approved the final version of the manuscript.

\section{Author Details}

1Department of Immunology, Faculty of Medicine Siriraj Hospital, Mahidol University, Bangkok 10700, Thailand, 2Department of Infectious and Tropical Diseases, London School of Hygiene and Tropical Medicine, London WC1E 7HT, UK and ${ }^{3}$ Division of Microbiology, Institute for Animal Health, Compton, Berkshire RG20 7NN, UK

Received: 18 August 2009 Accepted: 14 June 2010

Published: 14 June 2010

\section{References}

1. White NJ: Melioidosis. Lancet 2003, 361:1715-1722

2. Cheng AC, Currie BJ: Melioidosis: epidemiology, pathophysiology, and management. Clin Microbiol Rev 2005, 18:383-416.

3. Currie BJ, Jacups SP: Intensity of rainfall and severity of melioidosis, Australia. Emerg Infect Dis 2003, 9:1538-1542. 
4. Suputtamongkol Y, Hall AJ, Dance DA, Chaowagul W, Rajchanuvong A, Smith MD, White NJ: The epidemiology of melioidosis in Ubon Ratchatani, northeast Thailand. Int J Epidemio/ 1994, 23:1082-1090.

5. Leelarasamee A, Trakulsomboon S, Kusum M, Dejsirilert S: Isolation rates of Burkholderia pseudomallei among the four regions in Thailand. Southeast Asian J Trop Med Public Health 1997, 28:107-113.

6. Vuddhakul V, Tharavichitkul P, Na-Ngam N, Jitsurong S, Kunthawa B, Noimay P, Noimay P, Binla A, Thamlikitkul V: Epidemiology of Burkholderia pseudomallei in Thailand. Am J Trop Med Hyg 1999, 60:458-461.

7. Wongpokhom N, Kheoruenromne I, Suddhiprakarn A, Gilkes RJ: Micromorphological properties of salt affected soils in Northeast Thailand. Geoderma 2008, 144:158-170.

8. O'Quinn AL, Wiegand EM, Jeddeloh JA: Burkholderia pseudomallei kills the nematode Caenorhabditis elegans using an endotoxin-mediated paralysis. Cell Microbiol 2001, 3:381-393.

9. Vandamme P, Holmes B, Vancanneyt M, Coenye T, Hoste B, Coopman R, Revets $\mathrm{H}$, Lauwers S, Gillis M, Kersters K, et al:: Occurrence of multiple genomovars of Burkholderia cepacia in cystic fibrosis patients and proposal of Burkholderia multivorans sp. nov. Int J Syst Bacteriol 1997, 47:1188-1200.

10. Mahenthiralingam E, Baldwin A, Vandamme P: Burkholderia cepacia complex infection in patients with cystic fibrosis. J Med Microbiol 2002, 51:533-538.

11. Widdicombe $\mathrm{JH}$ : Altered $\mathrm{NaCl}$ concentration of airway surface liquid in cystic fibrosis. Pflugers Arch 2001, 443(Suppl 1):S8-10.

12. Joris L, Dab I, Quinton PM: Elemental composition of human airway surface fluid in healthy and diseased airways. Am Rev Respir Dis 1993, 148:1633-1637.

13. O'Carroll MR, Kidd TJ, Coulter C, Smith HV, Rose BR, Harbour C, Bell SC: Burkholderia pseudomallei: another emerging pathogen in cystic fibrosis. Thorax 2003, 58:1087-1091.

14. Choy JL, Mayo M, Janmaat A, Currie BJ: Animal melioidosis in Australia. Acta Trop 2000, 74:153-158.

15. Dance DA: Ecology of Burkholderia pseudomallei and the interactions between environmental Burkholderia spp. and human-animal hosts. Acta Trop 2000, 74:159-168.

16. Yamamoto T: [Stress response of pathogenic bacteria--are stress proteins virulence factors?]. Nippon Saikingaku Zasshi 1996, 51:1025-1036

17. Pumirat $P$, Saetun $P$, Sinchaikul $S$, Chen ST, Korbsrisate $S$, Thongboonkerd $\mathrm{V}$ : Altered secretome of Burkholderia pseudomallei induced by salt stress. Biochim Biophys Acta 2009, 1794:898-904.

18. Bhatt $\mathrm{S}$, Weingart $\mathrm{CL}$ : Identification of sodium chloride-regulated genes in Burkholderia cenocepacia. Curr Microbiol 2008, 56:418-422.

19. Aspedon A, Palmer K, Whiteley M: Microarray analysis of the osmotic stress response in Pseudomonas aeruginosa. J Bacteriol 2006, 188:2721-2725.

20. Walker KA, Miller VL: Regulation of the Ysa type III secretion system of Yersinia enterocolitica by YsaE/SycB and YsrS/YsrR. J Bacteriol 2004, 186:4056-4066.

21. Mildiner-Earley S, Walker KA, Miller VL: Environmental stimuli affecting expression of the Ysa type three secretion locus. Adv Exp Med Biol 2007, 603:211-216.

22. Stevens MP, Haque A, Atkins T, Hill J, Wood MW, Easton A, Nelson M, Underwood-Fowler C, Titball RW, Bancroft GJ, et al:: Attenuated virulence and protective efficacy of a Burkholderia pseudomallei bsa type III secretion mutant in murine models of melioidosis. Microbiology 2004, 150:2669-2676.

23. Warawa J, Woods DE: Type III secretion system cluster 3 is required for maximal virulence of Burkholderia pseudomallei in a hamster infection model. FEMS Microbiol Lett 2005, 242:101-108.

24. Stevens MP, Wood MW, Taylor LA, Monaghan P, Hawes P, Jones PW, Wallis TS, Galyov EE: An Inv/Mxi-Spa-like type III protein secretion system in Burkholderia pseudomallei modulates intracellular behaviour of the pathogen. Mol Microbiol 2002, 46:649-659.

25. Muangsombut V, Suparak S, Pumirat P, Damnin S, Vattanaviboon P, Thongboonkerd V, Korbsrisate S: Inactivation of Burkholderia pseudomallei bsaQ results in decreased invasion efficiency and delayed escape of bacteria from endocytic vesicles. Arch Microbiol 2008, 190:623-631.
26. Mizusaki H, Takaya A, Yamamoto T, Aizawa S: Signal pathway in saltactivated expression of the Salmonella pathogenicity island 1 type III secretion system in Salmonella enterica serovar Typhimurium. $J$ Bacteriol 2008, 190:4624-4631.

27. Haraga A, West TE, Brittnacher MJ, Skerrett SJ, Miller SI: Burkholderia thailandensis as a model system for the study of the virulenceassociated type III secretion system of Burkholderia pseudomallei. Infect Immun 2008, 76:5402-5411.

28. Stevens MP, Friebel A, Taylor LA, Wood MW, Brown PJ, Hardt WD, Galyov EE: A Burkholderia pseudomallei type III secreted protein, BopE, facilitates bacterial invasion of epithelial cells and exhibits guanine nucleotide exchange factor activity. J Bacterio/ 2003, 185:4992-4996.

29. Holden MT, Titball RW, Peacock SJ, Cerdeno-Tarraga AM, Atkins T, Crossman LC, Pitt T, Churcher C, Mungall K, Bentley SD, et al:: Genomic plasticity of the causative agent of melioidosis, Burkholderia pseudomallei. Proc Natl Acad Sci USA 2004, 101:14240-14245.

30. Rodrigues F, Sarkar-Tyson M, Sarah V, Harding SV, Siew Hoon Sim SH, Hui Hoon Chua HH, Lin CH, Han X, Krishna M, Karuturi RKM, Sung K, Yu K, et al:: Global Map of Growth-Regulated Gene Expression in Burkholderia pseudomallei, the Causative Agent of Melioidosis. J Bacterio/ 2006, 188:8178-8188.

31. Kimura Y, Ohtani M, Takegawa K: An adenylyl cyclase, CyaB, acts as an osmosensor in Myxococcus xanthus. J Bacteriol 2005, 187:3593-3598.

32. Nagarajan T, Vanderleyden J, Tripathi AK: Identification of salt stress inducible genes that control cell envelope related functions in Azospirillum brasilense Sp7. Mol Genet Genomics 2007, 278:43-51.

33. Zhang YX, Denoya CD, Skinner DD, Fedechko RW, McArthur HA, Morgenstern MR, Davies RA, Lobo S, Reynolds KA, Hutchinson CR: Genes encoding acyl-CoA dehydrogenase (AcdH) homologues from Streptomyces coelicolor and Streptomyces avermitilis provide insights into the metabolism of small branched-chain fatty acids and macrolide antibiotic production. Microbiology 1999, 145:2323-2334.

34. Mikami K, Murata N: Membrane fluidity and the perception of environmental signals in cyanobacteria and plants. Prog Lipid Res 2003, 42:527-543.

35. Suparak S, Kespichayawattana W, Haque A, Easton A, Damnin S, Lertmemongkolchai G, Bancroft GJ, Korbsrisate S: Multinucleated giant cell formation and apoptosis in infected host cells is mediated by Burkholderia pseudomallei type III secretion protein BipB. J Bacteriol 2005, 187:6556-6560.

36. Moore RA, Reckseidler-Zenteno S, Kim H, Nierman W, Yu Y, Tuanyok A, Warawa J, DeShazer D, Woods DE: Contribution of gene loss to the pathogenic evolution of Burkholderia pseudomallei and Burkholderia mallei. Infect Immun 2004, 72:4172-4187.

37. Korbsrisate S, Vanaporn M, Kerdsuk P, Kespichayawattana W, Vattanaviboon P, Kiatpapan P, Lertmemongkolchai G: The Burkholderia pseudomallei RpoE (AlgU) operon is involved in environmental stress tolerance and biofilm formation. FEMS Microbiol Lett 2005, 252:243-249.

38. Wu W, Badrane H, Arora S, Baker HV, Jin S: MucA-mediated coordination of type III secretion and alginate synthesis in Pseudomonas aeruginosa. J Bacterio/ 2004, 186:7575-7585.

39. Emerson JE, Stabler RA, Wren BW, Fairweather NF: Microarray analysis of the transcriptional responses of Clostridium difficile to environmental and antibiotic stress. J Med Microbiol 2008, 57:757-764.

doi: 10.1186/1471-2180-10-171

Cite this article as: Pumirat et al., Global transcriptional profiling of Burk-

holderia pseudomallei under salt stress reveals differential effects on the Bsa type III secretion system BMC Microbiology 2010, 10:171 